\title{
POR QUE TODOS OS NITRATOS SÃO SOLÚVEIS?
}

\author{
Luciana Almeida Silva, Cláudia Rocha Martins e Jailson Bittencourt de Andrade* \\ Instituto de Química, Universidade Federal da Bahia, Campus de Ondina, 40170-290 Salvador - BA
}

Recebido em 2/9/03; aceito em 20/5/04; publicado na web em 9/8/04

\begin{abstract}
WHY ARE ALL NITRATES SOLUBLE? The solubility rules presented in the majority of introductory texts of chemistry usually do not comprise a systematic analysis of the dissolution processes, neither from a microscopic nor from a macroscopic point of view. The solubility of nitrates in aqueous solution is discussed in this article, focusing on the thermodynamic data and the properties of the nitrate ion.
\end{abstract}

Keywords: solubility; nitrates; thermodynamic properties.

\section{INTRODUÇÃO}

O "desaparecimento" de uma substância quando misturada a outra é um interessante fenômeno que fascina cientistas há anos, além de despertar interesses econômicos e, até mesmo, de saúde pública. Um exemplo recente que abalou a sociedade brasileira foi a suspeita de contaminação de um contraste à base de $\mathrm{BaSO}_{4}$, usado em radioscopia e radiografia para destacar órgãos, que pode ter causado a morte de pelo menos 21 pessoas. O sulfato de bário é usado para este fim por ser um sal praticamente insolúvel em água $(0,0002 \mathrm{~g} / 100 \mathrm{~g}$ de água) e em soluções ácidas diluídas. Análises de amostras deste medicamento, produzido por um determinado fabricante, constataram a presença de $\mathrm{BaCO}_{3}$. Embora o carbonato de bário apresente também baixa solubilidade em água (0,002 g/100 g de água), este sal é solúvel em soluções ácidas diluídas como o suco gástrico, um fluido digestivo ácido que contém, entre outras substâncias, $\mathrm{HCl}$ (reação 1). Além disso, $\mathrm{BaCO}_{3}$, em presença de $\mathrm{CO}_{2} \mathrm{e}_{2} \mathrm{O}$, forma bicarbonato de bário, que é um sal aquossolúvel (reação 2).

$$
\begin{array}{ll}
\mathrm{BaCO}_{3}(\mathrm{~s})+2 \mathrm{H}^{+}(\mathrm{aq}) \rightarrow \mathrm{Ba}^{2+}(\mathrm{aq})+\mathrm{CO}_{2}(\mathrm{~g})+\mathrm{H}_{2} \mathrm{O}(\mathrm{l}) & (\text { reação 1) } \\
\mathrm{BaCO}_{3}(\mathrm{~s})+\mathrm{CO}_{2}(\mathrm{~g})+\mathrm{H}_{2} \mathrm{O}(\mathrm{l}) \rightarrow \mathrm{Ba}\left(\mathrm{HCO}_{3}\right)_{2}(\mathrm{aq}) & (\text { reação 2) }
\end{array}
$$

Como todos os compostos de bário solúveis em água ou ácidos são venenosos ${ }^{1}, \mathrm{o} \mathrm{BaCO}_{3}$ apresenta efeito deletério à saúde humana. Esse caso ilustra bem a importância do conhecimento da solubilidade, pois o engano com relação ao meio reacional pode ter sido o motivo que levou várias pessoas à morte.

\section{SOLUBILIDADE}

Solubilidade, por definição, é a concentração de soluto dissolvido em um solvente em equilíbrio com o soluto não dissolvido à temperatura e pressão especificadas, ou seja, é a medida da quantidade máxima de soluto que pode ser dissolvida em um determinado solvente. O tamanho molecular (ou iônico), a polaridade (ou carga), forças dispersivas e dipolares, ligações de hidrogênio e a temperatura são fatores que se destacam na determinação da solubilidade e devem ser considerados no seu entendimento. Entretanto, é comum encontrar em livros textos de química destinados ao ensino médio, e em vários outros dedicados ao ensino superior, tabelas de regras de

*e-mail: jailsong@ufba.br

solubilidade (o tipo mais freqüente é mostrado na Tabela 1) que apresentam uma série de compostos iônicos, classificando-os simplesmente como solúveis ou insolúveis em água. Normalmente, tais regras não vêm acompanhadas de uma análise sistemática do processo de dissolução, tanto do ponto de vista microscópico quanto do ponto de vista macroscópico.

Com o objetivo de contribuir para a correção desta falha, iniciamos uma discussão em torno de afirmações muito comuns, porém inconsistentes, como todos os nitratos são solúveis, enquanto a maioria dos carbonatos apresenta baixa solubilidade em água.

Tabela 1. Regras de solubilidade para compostos iônicos em água a $298 \mathrm{~K}$

\section{Compostos solúveis}

Quase todos os sais de $\mathrm{Na}^{+}, \mathrm{K}^{+}, \mathrm{NH}_{4}^{+}$

Haletos: sais de $\mathrm{Cl}^{-}, \mathrm{Br}^{-}$e I

Fluoretos

Sais de $\mathrm{NO}_{3}^{-}, \mathrm{ClO}_{3}^{-}, \mathrm{ClO}_{4}^{-}, \mathrm{C}_{2} \mathrm{H}_{3} \mathrm{O}_{2}^{2-}$

Sulfatos

Ácidos inorgânicos

Compostos insolúveis

Sais de $\mathrm{CO}_{3}^{2-}, \mathrm{PO}_{4}^{3-}, \mathrm{C}_{2} \mathrm{O}_{4}^{2-}$ e $\mathrm{CrO}_{4}{ }^{2-}$

Sulfetos

Hidróxidos e óxidos metálicos

\section{ENTALPIA DE DISSOLUÇÃO}

Para que um composto iônico se dissolva em um dado solvente, a atração eletrostática entre os íons no retículo deve ser superada. Entretanto, a idéia de dissociação de sais em partículas carregadas, a princípio, poderia ser um contra-senso por conta das fortes interações interiônicas. Por exemplo, se tentássemos separar completamente os
Exceções

Sais de $\mathrm{NH}_{4}^{+}$e de cátions de metais alcalinos Sais de $\mathrm{NH}_{4}^{+}, \mathrm{Ca}^{2+}, \mathrm{Sr}^{2+} \mathrm{e}$ de cátions de metais alcalinos Hidróxidos e óxidos de $\mathrm{Ca}^{2+}, \mathrm{Sr}^{2+}, \mathrm{Ba}^{2+}$ e os cátions de metais alcalinos 
íons sódio dos íons cloreto em um mol de $\mathrm{NaCl}$, a uma distância de $10 \mathrm{~cm}$, seria necessário exercer uma força de aproximadamente $10^{14}$ $\mathrm{t}$ - $1 \mathrm{tf}$ (tonelada força) equivale a $9810 \mathrm{~N}$ na superfície terrestre. $\mathrm{E}$ caso as partículas positivas ou negativas desse sal fossem removidas para a superfície da lua, ainda assim, cátions e ânions se atrairiam com uma força de cerca de meia tonelada ${ }^{11}$.

Então, que efeitos podem operar em oposição a essas poderosas forças para permitir a existência de soluções iônicas? Podem-se considerar três como os principais: desordem, permissividade do meio e energia de interação entre moléculas do solvente e os íons do soluto. Desses, o último é o mais importante. Por isso dedicaremos esta seção a sua discussão detalhada.

A capacidade de dissolução de um sal em água é fortemente influenciada pela entalpia de dissolução, que representa o calor envolvido na dissolução de uma substância em um dado solvente à pressão constante, e pode ser determinada pelo balanço energético das etapas teóricas envolvidas na formação da solução. A energia resultante nesse processo pode ser calculada por:

\section{$\Delta_{\text {sol }} H=\Delta H($ soluto-solvente $)-\Delta H($ soluto-soluto $)-$ $\Delta H$ (solvente-solvente)}

No processo de solvatação, grandes perturbações nas interações solvente-solvente ocorrerão, modificando significativamente a sua estrutura nas adjacências do íon. Solventes que interagem fortemente com íons apresentam forças intermoleculares intensas, tais como interações dipolares e ligações de hidrogênio. Entretanto, se compararmos a intensidade das interações solvente-solvente (dipolo-dipolo), soluto-solvente (íon-dipolo) e soluto-soluto (íon-íon), concluiremos facilmente que a atração eletrostática é mais intensa entre partículas carregadas, ou seja, íon-íon. Porém, existe um elevado número de interações íon-dipolo atuando em cada íon. Como resultado, a energia de interação soluto-solvente torna-se grosseiramente da mesma ordem de grandeza da energia de coesão entre cátions e ânions, enquanto que as interações solvente-solvente tornam-se negligenciáveis nas adjacências dos íons comparadas à magnitude dessas.

No Esquema 1 está representado, através do ciclo de Born-Haber, o processo de dissolução de um sal. Considerando que as interações solvente-solvente neste caso são negligenciáveis, a energia resultante do processo de dissolução $\left(\Delta_{s o l} H ; H=\right.$ entalpia $)$ será o somatório das energias envolvidas em duas etapas:

i) uma é o processo desfavorável de separação "infinita" dos íons do retículo que corresponde à entalpia de rede $\left(\Delta H_{\text {rede }}\right)$, definida como a entalpia de reação para a formação de um gás de íons a partir do sólido cristalino (reação 3 ) $^{2-5}$;

$\mathrm{MX}(\mathrm{s}) \rightarrow \mathrm{M}^{+}(\mathrm{g})+\mathrm{X}^{-}(\mathrm{g})$

(reação 3)

A definição de energia de rede como energia requerida não é uniforme na maioria dos textos destinados a disciplinas introdutórias de química e até mesmo em livros de química avançada ${ }^{2-9}$. Muitos deles definem energia de rede como a energia liberada quando o cristal é formado a partir dos íons gasosos separados a uma distância infinita ${ }^{6}$ 9 . Essa falta de consenso pode gerar dúvidas conceituais e dificultar o entendimento. Embora a terminologia química adotada nesse texto seja a recomendada pela IUPAC, o termo Lattice Energy não consta na lista da "IUPAC Compendium of Chemical Terminology"10, por isso adotamos a definição encontrada em referências mais recentes;

ii) a outra etapa envolve o processo de solvatação dos íons $\left(\Delta_{\text {solv }} H\right)$, que é energeticamente favorável.

Deste modo, a variação de entalpia na dissolução de um sal pode ser obtida com uma boa aproximação empregando o ciclo de BornHaber (Esquema 1).

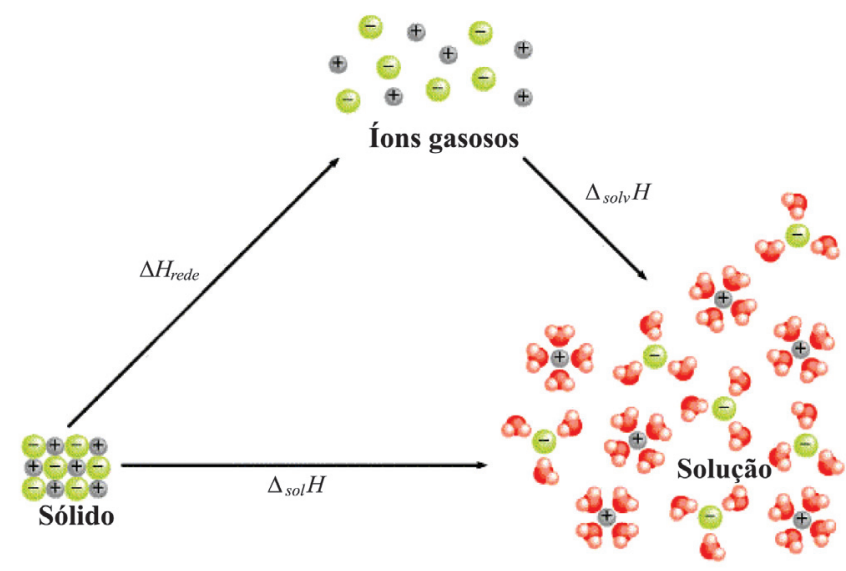

Esquema 1. Ciclo de Born-Haber para dissolução de um sal

O Esquema 2 ilustra um processo de dissolução exotérmica. Nesta situação, a energia de rede é superada pela entalpia de solvatação. As interações soluto-soluto (íon-íon) e solvente-solvente (dipolo-dipolo) são menos intensas que o somatório de todas as interações soluto-solvente (íon-dipolo). A entalpia de dissolução será negativa $\left(\Delta_{s o l} H<0\right)$. Macroscopicamente registra-se um aumento na temperatura. Processos desta natureza tendem a ser espontâneos, uma vez que transformações, sejam físicas ou químicas, ocorrerão espontaneamente no

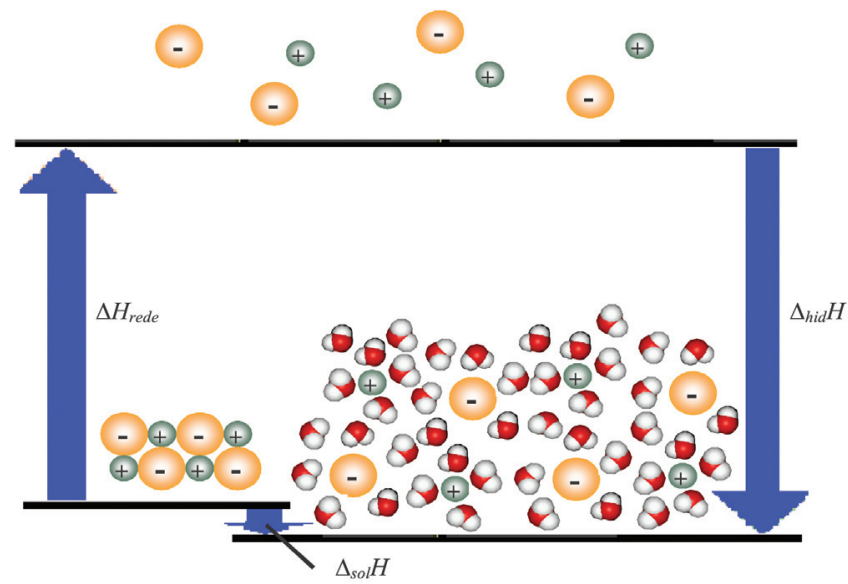

Esquema 2. Processo de dissolução exotérmica

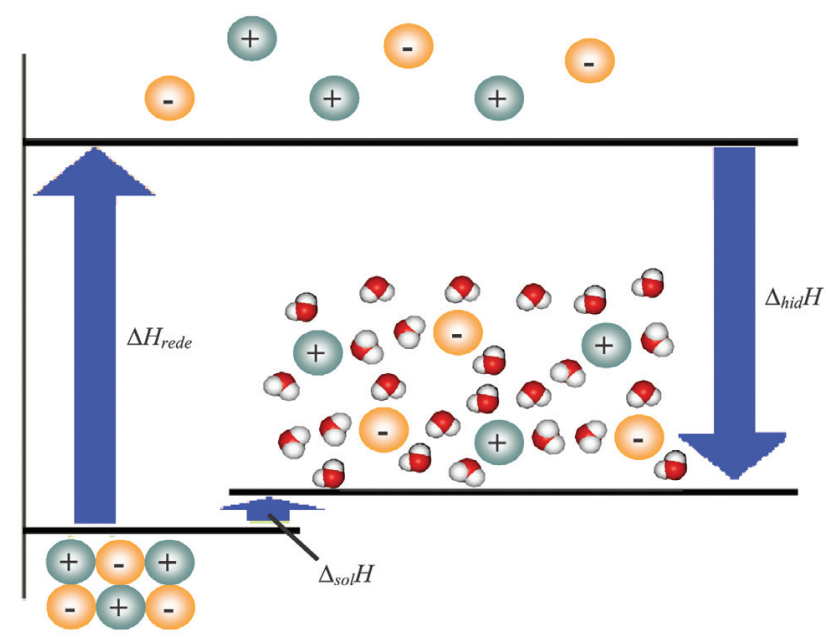

Esquema 3. Processo de dissolução endotérmica 
sentido de diminuição da energia livre ( $\Delta G=\Delta H-T \Delta S<0$; o segundo termo da equação, relacionado à desordem, será discutido na próxima seção).

Em muitos casos, a entalpia de dissolução para compostos iônicos em água é positiva $\left(\Delta_{s o l} H>0\right)$. O Esquema 3 mostra que somente a entalpia de solvatação não é suficiente para superar as forças atrativas entre os íons. Quando isso ocorre, a solução é forçada a realizar trabalho $(w)$ para separá-los, gastando energia do próprio sistema (energia interna $-U)$ num processo adiabático $(\Delta U=w)$. Nestes casos, registra-se uma diminuição de temperatura. Se a entalpia de solução for muito positiva e o segundo termo da equação de energia livre (relacionado à desordem) não for suficiente para compensá-la $(\Delta G=\Delta H-T \Delta S>0)$, o composto iônico será insolúvel.

\section{ENTROPIA}

Embora a capacidade de dissolução de um sal seja fortemente afetada pela entalpia de dissolução, somente este parâmetro não define a sua solubilidade. Para muitos sais, a contribuição do fator entrópico no processo de dissolução é comparável à entalpia de solvatação. Um aumento da desordem é esperado quando os íons deixam o sólido e passam para a solução; em contrapartida, há uma reorganização das moléculas do solvente com a solvatação desses íons. Embora seja possível medir a entropia de um soluto em uma solução eletrolítica, não existe um meio experimental de atribuir entropias a cátions e ânions separadamente, uma vez que é impossível ter uma solução só de cátions ou só de ânions. Por isso, as entropias de íons hidratados são medidas em relação à entropia padrão de íons $\mathrm{H}^{+}$em água, estabelecida como zero em qualquer temperatura $\left[\mathrm{S}^{\circ}\right.$ $\left(\mathrm{H}^{+}, \mathrm{aq}\right)=0$ ]. Deste modo, a entropia de íons hidratados provenientes da dissociação do sal também pode ser positiva ou negativa. Quando a entropia é positiva, a organização das moléculas de água em torno do íon é menor que em torno do íon $\mathrm{H}^{+}$. Já se a entropia for negativa, a organização das moléculas de água em torno do íon será maior que em torno do íon $\mathrm{H}^{+}$.

\section{EFEITO DA CARGA E DO RAIO DOS ÍONS}

A carga e o tamanho dos íons exercem influência significativa na energia de rede e na energia de solvatação. A Equação 1 representa a energia total para um mol de um cristal em função de $r$, considerando as contribuições devidas à atração eletrostática (primeiro termo) e à repulsão na superposição das nuvens eletrônicas dos íons (segundo termo). Nesta equação, a dependência da carga e do tamanho dos íons é evidenciada:

$E=\frac{A N Z^{+} Z^{-} e^{2}}{4 \pi \varepsilon_{o} r}+\frac{N B}{r^{n}}$

$E=$ energia total em um mol de um cristal

$Z^{+}$e $Z=$ número de carga dos íons

$e=$ carga do elétron $=1,6 \times 10^{-19} \mathrm{C}$

$\varepsilon_{o}=$ permissividade do vácuo $=8,85 \times 10^{-12} \mathrm{C}^{2} \mathrm{~J}^{-1} \mathrm{~m}^{-1}$

$r=$ distância de separação dos íons

$A=$ constante de Madelung

$N=$ constante de Avogadro

$B=$ constante de repulsão

$n=$ expoente de Born

A informação do expoente de Born, $n$, pode ser obtida experimentalmente por dados de compressibilidade, que é a medida da resistência que os íons exibem quando são forçados a uma grande aproximação. A constante de Madelung $(A)$ é um número adimencional relacionado às características geométricas do sólido.
A energia será mínima (energia de rede $-E_{r}$ ) quando a distância de equilíbrio de separação dos íons é alcançada $\left(r=r_{o}\right)$. Neste caso, a Equação 1 pode ser escrita como (veja detalhes nas referências 7-9):

$E_{r}=-\frac{A N Z^{+} Z^{-} e^{2}}{4 \pi \varepsilon_{o} r_{o}}\left(1-\frac{1}{n}\right)$

O valor da energia de rede será maior quando os íons forem similares em tamanho, especialmente se forem pequenos e com carga elevada. A presença de cátions muito maiores que ânions e viceversa, pode reduzir o valor de $E_{r}$.

A energia de solvatação total é a soma das energias de solvatação dos dois íons individuais e está diretamente relacionada à habilidade inerente do solvente para coordenar os íons envolvidos e à natureza desses íons. A atração coulombiana entre um par de íons é inversamente proporcional à permissividade do meio:

$E=\frac{Z^{+} Z^{-} e^{2}}{4 \pi \varepsilon r}$

$E=$ energia eletrostática entre um par de íons

$Z^{+}$e $Z=$ números de carga dos íons

$\varepsilon=$ permissividade do meio

$r=$ distância de separação dos íons

A permissividade relativa $\left(\varepsilon / \varepsilon_{o}\right)$ para espaços livres (vácuo) é 1,00; já para solventes polares, esses valores são consideravelmente maiores. Nas soluções de íons solvatados por um solvente com alta permissividade relativa (e.g. $\varepsilon_{\text {agua }} / \varepsilon_{o}=81,7$; neste caso o termo usual é hidratação), a força de atração entre cátions e ânions será apreciavelmente menor devido ao poder de "blindagem" do solvente, o que favorece o processo de dissolução. Além disso, quanto mais polar for o solvente, maior será sua capacidade de se coordenar ao íon. Por outro lado, quanto menor for o íon e maior a carga, tanto maiores serão a força e o número de interações com as moléculas do solvente.

O efeito desses parâmetros (tamanho e carga dos íons) também pode ser evidenciado na entropia de íons solvatados. Íons grandes com carga pequena contribuem favoravelmente para o processo de dissolução, enquanto que para íons muito pequenos com carga elevada, a contribuição do fator entrópico é desfavorável. A forte interação do íon com o dipolo da água induz uma maior organização das moléculas de água em torno do íon e, com isso, a desordem da solução diminui mais do que no caso de íons grandes com pequena carga.

\section{SOLUBILIDADE DE NITRATOS}

Com relação à questão inicial, por que todos os nitratos são solúveis? a melhor maneira de avaliar o comportamento de nitratos frente à água é analisar as propriedades físico-químicas deste ânion. O nitrato é um íon poliatômico que apresenta carga pequena, -1 , e raio iônico de 165 pm (Tabela 2). Essas características lhe conferem uma baixa entalpia de hidratação $\left(\Delta_{\text {hid }} \mathrm{H}^{\circ}=-295 \mathrm{~kJ} \mathrm{~mol}^{-1}\right)$ e entropia relativamente elevada em água $\left(\mathrm{S}^{\circ}=146,7 \mathrm{~J} \mathrm{~K}^{-1} \mathrm{~mol}^{-1}\right)$. Se compararmos com outros íons poliatômicos isoeletrônicos, como por exemplo, o $\mathrm{CO}_{3}^{2-}$, constataremos que a entropia deste íon hidratado é consideravelmente menor $\left(\mathrm{S}^{\circ}=-50,0 \mathrm{~J} \mathrm{~K}^{-1} \mathrm{~mol}^{-1}\right)$. Embora o íon carbonato tenha raio (164 pm) muito próximo ao do íon nitrato, a carga é duas vezes maior. A elevada relação carga/raio aumenta a energia reticular dos carbonatos que é compensada pelo aumento da energia de hidratação deste ânion $\left(\Delta_{\text {hid }} \mathrm{H}^{\circ}=-1120 \mathrm{~kJ} \mathrm{~mol}^{-1}\right)$. Por outro lado, a alta afinidade pela água resulta numa maior organização das moléculas de água em torno deste íon comparada ao íon $\mathrm{NO}_{3}^{-}$hidratado, 
o que desfavorece o processo de dissolução do ponto de vista entrópico.

Sais formados por associação do íon nitrato com cátions univalentes pequenos apresentam energias de rede baixas. Na dissolução do $\mathrm{LiNO}_{3}$, por exemplo, a entalpia de hidratação do sal é suficiente para superar a entalpia de rede, por ter o íon $\mathrm{Li}^{+}$relação carga/

Tabela 2. Raio iônico e entropia de alguns ânions

\begin{tabular}{lcc}
\hline Ânion & $\mathrm{r}_{\text {ânion }} / \mathrm{pm}^{\text {(a) }}$ & $\begin{array}{c}\mathrm{S}^{\circ} / \mathrm{J}^{-1} \mathrm{Kol}^{-1} \\
\text { (ânion hidratado) }\end{array}$ \\
\hline $\mathrm{F}^{-}$ & 136 & $-13,8$ \\
$\mathrm{Cl}^{-}$ & 181 & 56,6 \\
$\mathrm{Br}$ & 195 & 82,6 \\
$\mathrm{I}^{-}$ & 216 & 106,5 \\
$\mathrm{OH}^{-}$ & 119 & $-10,9$ \\
$\mathrm{HCO}_{3}^{-}$ & 142 & 98,4 \\
$\mathrm{CH}_{3} \mathrm{CO}_{2}^{-}$ & 148 & 87,0 \\
$\mathrm{CO}_{3}^{2-}$ & 164 & $-50,0$ \\
$\mathrm{NO}_{3}^{-}$ & 165 & 146,7 \\
$\mathrm{ClO}_{4}^{-}$ & 226 & 184,0 \\
$\mathrm{SO}_{4}{ }^{-2}$ & 244 & 18,5 \\
\hline
\end{tabular}

Fontes: (a) ref. 9; (b) ref. 12 e 13.

Tabela 3. Propriedades termodinâmicas de alguns cátions

\begin{tabular}{lccc}
\hline Cátion & $\mathrm{r}_{\text {cátion }} / \mathrm{pm}^{\text {(a) }}$ & $\Delta_{\text {hid }} \mathrm{H}^{\mathrm{o}} / \mathrm{kJ} \mathrm{mol}^{-1(\mathrm{~b})}$ & $\begin{array}{c}\mathrm{S}^{\circ} / \mathrm{J} \mathrm{K}^{-1} \mathrm{~mol}^{-1} \\
\text { (cátion hidratado) (b) }\end{array}$ \\
\hline $\mathrm{Li}^{+}$ & 78 & -558 & 12,2 \\
$\mathrm{Na}^{+}$ & 98 & -444 & 59,0 \\
$\mathrm{~K}^{+}$ & 138 & -361 & 101,2 \\
$\mathrm{Rb}^{+}$ & 149 & -305 & 121,8 \\
$\mathrm{Cs}^{+}$ & 165 & -289 & 132,1 \\
$\mathrm{NH}_{4}^{+}$ & 151 & -355 & 111,2 \\
$\mathrm{Ag}^{+}$ & 113 & -510 & 73,5 \\
$\mathrm{Mg}^{2+}$ & 78 & -2003 & $-138,1$ \\
$\mathrm{Ca}^{2+}$ & 106 & -1657 & $-56,2$ \\
$\mathrm{Sr}^{2+}$ & 127 & -1524 & $-9,6$ \\
$\mathrm{Ba}^{2+}$ & 136 & -1390 & 9,6 \\
\hline
\end{tabular}

Fontes: (a) Tabela periódica editada pela SBQ, exceto para $\mathrm{NH}_{4}^{+}$, extraído da ref. 9; (b) ref. 12 e 13. raio relativamente elevada comparado aos demais cátions dos metais alcalinos, resultando num processo de dissolução exotérmico (Tabelas 3 e 4). Em se tratando de cátions pequenos com carga elevada, há um expressivo aumento na energia de rede que, porém, é acompanhado de um aumento substancial na energia de hidratação (Tabelas 3 e 4), uma vez que íons com elevada relação carga/raio têm grande afinidade pelo dipolo da molécula de água. Mesmo que a hidratação de cátions com relação carga/raio elevada seja acompanhada por uma diminuição de entropia, a entropia do íon nitrato hidratado, normalmente, é suficiente para compensá-la. A contribuição entálpica, neste caso, é reforçada pela entropia de dissolução, resultando em uma energia livre favorável à dissolução (Tabela 4).

Por outro lado, é incomum a formação de sais inorgânicos com cátions muito maiores que o íon nitrato. Já quando o sal é formado por combinação com cátions univalentes que possuem raios mais próximos ao raio do $\mathrm{NO}_{3}^{-}$(ex. $\mathrm{CsNO}_{3}$ - Tabela 3), era de se esperar um aumento na energia de rede, favorecido pelo fator geométrico. Entretanto, o ânion em questão é um íon poliatômico, o que destoa bastante do modelo ideal de esferas rígidas, e o observado, nestes casos, é uma sensível diminuição da energia de rede devida à maior distância interiônica. Para sais desse tipo, é possível que a baixa entalpia de hidratação (soma das entalpias de hidratação de cátions e ânions individuais) não seja suficiente para superar a energia de rede. Os processos de dissolução nestes casos são endotérmicos (Tabela 4), ou seja, a solução consome energia interna na separação dos íons. Para compensar o custo energético, o fator entrópico deve ser pronunciado, o que de fato acontece, pois cátions com raios muito grandes e cargas pequenas terão baixa entalpia de hidratação, porém alta entropia. A entropia de hidratação do cátion e do ânion é suficiente para compensar a baixa entalpia de hidratação, favorecendo a solubilidade.

A variação de energia livre na dissolução de nitratos normalmente é negativa, determinando a espontaneidade do processo (Tabela 4). No entanto, podem-se perceber alguns valores de $\Delta_{\text {sol }} \mathrm{G}^{\circ}$ positivos. Os processos de dissolução destes sais são endotérmicos, o que significa dizer que as reações de precipitação são exotérmicas, porém a precipitação é entropicamente desfavorável. Considerando que os íons devem abandonar um meio onde a entropia é elevada para se agregarem e retornarem à forma cristalina, a energia livre de precipitação envolvida é muito pequena quando confrontados os dois termos $\left(\Delta_{\mathrm{ppt}} \mathrm{H}^{\circ} \mathrm{e}-\mathrm{T} \Delta_{\mathrm{ppt}} \mathrm{S}^{\mathrm{o}}\right.$; processo inverso à dissolução), o que permite um certo grau de solubilidade a estes sais. Por exemplo, o produto de solubilidade $\left(K_{p s}=e^{-\frac{\Delta_{s o l} G^{o}}{R T}}\right)$ de um dos nitratos menos solú-

Tabela 4. Dados termodinâmicos e solubilidade de alguns nitratos $(\mathrm{T}=298 \mathrm{~K})$

\begin{tabular}{|c|c|c|c|c|c|}
\hline Sal & $\Delta \mathrm{H}_{\text {rede }}^{\mathrm{o}} / \mathrm{kJ} \mathrm{mol}^{-1}$ & $\Delta_{\mathrm{sol}} \mathrm{H}^{\circ} / \mathrm{kJ} \mathrm{mol}^{-1}$ & $-\mathrm{T} \Delta_{\mathrm{sol}} \mathrm{S}^{\circ} / \mathrm{kJ} \mathrm{mol}^{-1}$ & $\Delta_{\mathrm{sol}} \mathrm{G}^{\mathrm{o}} / \mathrm{kJ} \mathrm{mol}^{-1}$ & $\begin{array}{c}\text { Solubilidade/ } \\
\mathrm{g} \text { de sal/100 } \mathrm{g} \mathrm{de} \mathrm{H}_{2} \mathrm{O}\end{array}$ \\
\hline $\mathrm{LiNO}_{3}$ & 848 & $-2,5$ & $-20,9$ & $-23,4$ & 102,0 \\
\hline $\mathrm{NaNO}_{3}$ & 755 & 20,5 & $-26,9$ & $-6,4$ & 91,2 \\
\hline $\mathrm{KNO}_{3}$ & 678 & 34,9 & $-34,5$ & 0,4 & 38,3 \\
\hline $\mathrm{RbNO}_{3}$ & 658 & 36,5 & $-35,9$ & 0,6 & 65,0 \\
\hline $\mathrm{CsNO}_{3}$ & 625 & 40,0 & $-37,0$ & 3,0 & 27,9 \\
\hline $\mathrm{NH}_{4} \mathrm{NO}_{3}$ & 676 & 25,7 & $-32,4$ & $-6,7$ & 212,5 \\
\hline $\mathrm{AgNO}_{3}$ & 822 & 22,6 & $-23,3$ & $-0,7$ & 234,4 \\
\hline $\mathrm{Mg}\left(\mathrm{NO}_{3}\right)_{2}$ & 2503 & $-90,9$ & 2,7 & $-88,2$ & 71,2 \\
\hline $\mathrm{Ca}\left(\mathrm{NO}_{3}\right)_{2}$ & 2228 & $-19,2$ & $-13,8$ & $-33,0$ & 143,9 \\
\hline $\mathrm{Sr}\left(\mathrm{NO}_{3}\right)_{2}$ & 2132 & 17,7 & $-19,5$ & $-1,8$ & 80,2 \\
\hline $\mathrm{Ba}\left(\mathrm{NO}_{3}\right)_{2}$ & 2016 & 35,7 & $-26,4$ & 9,3 & 10,3 \\
\hline
\end{tabular}

Notas: Os valores de $\Delta \mathrm{H}^{\circ}{ }_{\text {rede }}$ foram determinados experimentalmente de acordo com o ciclo de Born-Haber; $\Delta_{\text {sol }} \mathrm{S}^{\circ}=\mathrm{S}_{\text {aq }}^{\circ}-\mathrm{S}_{\mathrm{c}}^{\circ}\left(\mathrm{S}_{\text {aq }}^{\circ}=\right.$ entropia padrão dos íons em solução aquosa; $\mathrm{S}_{\mathrm{c}}{ }_{\mathrm{c}}=$ entropia padrão do cristal).

Fontes: todos os dados foram extraídos das ref. 12 e 13. 
veis, o $\mathrm{Ba}\left(\mathrm{NO}_{3}\right)_{2}$, é $4,6 \times 10^{-3}$, muito superior ao de sais tipicamente insolúveis em água, como por exemplo, o $\mathrm{BaSO}_{4}\left(\mathrm{~K}_{\mathrm{ps}}=1,1 \times 10^{-10}\right)$ e o $\mathrm{BaCO}_{3}\left(\mathrm{~K}_{\mathrm{ps}}=2,6 \times 10^{-9}\right)$.

\section{CONCLUSÕES}

Em geral, sais formados por cátions e ânions com tamanhos substancialmente diferentes são solúveis devido à baixa energia de rede.

A solubilidade de sais em água também aumenta com o incremento do tamanho de cátions ou ânions, devido a uma variação de entropia mais favorável na solvatação. Entretanto, o efeito da carga do íon é mais importante que o efeito do tamanho. A entropia de íons pequenos hidratados é pouco favorável para a dissolução, especialmente se a carga é elevada. Sais formados por íons de carga baixa, geralmente, são solúveis (ex.: sais de metais alcalinos, de $\mathrm{ClO}_{4}^{-}, \mathrm{NO}_{3}^{-}$, $\mathrm{C}_{2} \mathrm{H}_{3} \mathrm{O}_{2}^{-}$e haletos). Existem poucos sais solúveis de ânions de carga elevada, a menos que estejam associados a cátions univalentes.

Os sais de ânions com relação carga/raio reduzida, como é o caso dos nitratos, mesmo combinados com cátions pequenos de carga elevada, são solúveis, uma vez que a variação de entropia resultante da solvatação do ânion, associada à entalpia de solvatação do sal, normalmente, é suficiente para compensar o aumento na energia de rede e diminuição de entropia na solvatação de cátions com relação carga/raio elevada.

\section{AGRADECIMENTOS}

Ao CNPq, à FINEP e à FAPESB e aos assessores de Química Nova pelas valiosas sugestões.

\section{REFERÊNCIAS}

1. Gosselin, R. E.; Smith, R. P.; Hodge, H. C.; Clinical Toxicology of Commercial Products, $5^{\text {th }}$ ed., Williams \& Wilkins: Baltimore, 1984.

2. Douglas, B.; McDaniel, D.; Alexander, J.; Concepts and Models of Inorganic Chemistry, $3^{\text {rd }}$ ed., John Wiley \& Sons, Inc.: New York, 1994.

3. Atkins, P. W.; Physical Chemistry; $6^{\text {th }}$ ed., Oxford University Press: Oxford, 1998.

4. Atkins, P. W.; Jones, L. L.; Chemistry: molecules, matter and change, $3^{\text {rd }}$ ed., Freeman: New York, 1997.

5. Russel, J. B.; Química Geral, 2ª ed., Makron Books: São Paulo, 1994 vol. 1.

6. Brady, J. E.; Humiston, G. E.; Química Geral, $2^{a}$ ed., Livros Técnicos e Científicos Editora S. A.: Rio de Janeiro, 1986, vol. 1.

7. Duarte, A.D.; Cadernos Temáticos de Química Nova na Escola - Estrutura da Matéria: uma visão molecular, 2001, 4, 14

8. Lee, J. D.; Química Inorgânica não tão Concisa, tradução da $5^{\mathrm{a}}$ ed. inglesa, Ed. Edgard Blücher: São Paulo, 1999.

9. Huheey, J. E.; Keiter, E. A.; Keiter, R. L.; Inorganic Chemistry: Principles of Struture and Reactivity, $4^{\text {th }}$ ed., Harper Collins College Publishers: New York, 1993.

10. http://www.iupac.org/publications/compendium/S.html, acessada em Fevereiro 2004

11. Blackburn, T. R.; Equilibrium - A Chemistry of Solutions, $1^{\text {st }}$ ed., Holt Rinehart Winston: New York, 1969.

12. Lide, D. R., editor-in-chief; Handbook of Chemistry and Physics, $78^{\text {th }}$ ed., CRC Press, Inc: Cleveland, 1997 - 1998.

13. http://www.codata.org/databases/key1.html, acessada em Agosto 2003.

14. Letcher, T. M.; Battino, R.; J. Chem. Educ. 2001, 78, 103.

15. van der Sluys; W. G.; J. Chem. Educ. 2001, 78, 111.

16. http://www.iupac.org/reports/provisional/abstract02/ribeiro-dasilva_prs.pdf, acessada em Agosto 2003. 